\title{
Facile synthesis of nanostructured WO3 thin films and their characterization for ethanol sensing.
}

\begin{abstract}
A simple technique to fabricate nanostructured WO3 thin films onto conductomeric transducers has been employed for ethanol sensing application. Initially, pure tungsten (W) thin films were deposited onto the substrate employing RF sputterer and followed by an etching process. Three types of etching agent were used: nitric (HNO3), sulphuric (H2SO4), and phosphoric (H3PO4) acid. It was found that the surface morphology and crystallinity of the WO3 films were heavily dependant to the etchants employed during the fabrication process. The developed sensors were tested towards ethanol vapor of different concentrations (10-200 ppm) at temperatures between room and $450{ }^{\circ} \mathrm{C}$. The sensors showed stable and reproducible response at optimum operating temperatures. High sensor response towards vaporized ethanol as well as fast $\tau$ res and $\tau$ rec was observed during the "adsorption" and "desorption" interval. The recorded maximum response for these devices when exposed towards $100 \mathrm{ppm}$ ethanol was measured to be $8(\mathrm{Ro}=4.6 \mathrm{k} \Omega), 5.8$ $(\mathrm{Ro}=22.5 \mathrm{G} \Omega)$, and $5(\mathrm{Ro}=0.29 \mathrm{M} \Omega)$ for $\mathrm{HNO} 3$, H3PO4, and H2SO4, respectively. The optimum operating temperatures were determined to be $400,300-380$, and $360{ }^{\circ} \mathrm{C}$ for the sensors developed using $\mathrm{HNO} 3, \mathrm{H} 3 \mathrm{PO} 4$, and $\mathrm{H} 2 \mathrm{SO} 4$, respectively.
\end{abstract}

Keyword: Nanostructures, Thin films, Chemical synthesis, Sputtering, Electrical characterisation, Electrical properties 